\title{
Innovación docente en Psicología de la Atención y de la Percepción
}

\section{Teaching innovation in Psychology of Attention and Perception}

JuAn Pedro Vargas Romero

Universidad de Sevilla

Departamento de Psicología Experimental

vargas@us.es

ORCID: https://orcid.org/0000-0002-4358-5737

DOI: http://dx.doi.org/10.12795/9788447231003.088

Pp.: 1911-1927 


\section{Contexto de la intervención}

El actual título de Grado en Psicología se implantó en el curso 2009-2010. En la estructura del título, la asignatura "Psicología de la Atención y de la Percepción" pertenece al bloque de formación básica del primer cuatrimestre del primer curso. Los contenidos están estructurados en dos bloques que comprenden los contenidos de psicología de la percepción, por un lado y los de psicología de la atención por otro. Las clases suelen tener unos 55-65 alumnos de los que empiezan asistiendo la casi totalidad y al final del curso asisten una media de unos 35-40. La metodología usada hasta ahora iba en función del tipo de clase. Hasta el curso 2017-18, la asignatura se organizaba en función del número de alumnos de cada grupo de actividad. En las clases de grupo grande a la que asistían todos los alumnos, impartíamos teoría mediante clase magistral. En grupos medianos compuestos de la mitad de los alumnos, tratábamos temas monográficos mediante seminarios guiados. En grupos pequeños, donde asistía la cuarta parte de los alumnos hacíamos experimentos y redactábamos informes. El curso 2018-19, la asignatura cambió su estructura incrementando el número de clases de grupo grande y de grupo pequeño a cambio de la desaparición de las de grupo mediano. Esta fue la excusa o el motivo por el que empezamos a modificar la metodología de las clases de grupo grande para que fuesen más útiles. En el presente curso, debido a las medidas de distanciamiento social impuestas como consecuencia de la pandemia por enfermedad de coronavirus, las clases de grupo grande se impartirán de forma no presencial y las de grupo pequeño se impartirán de forma presencial.

Ciclos de Mejora en el Aula (2020). Experiencias de Innovación Docente de la US Esta obra se distribuye con la licencia Creative Commons 


\section{Diseño previo del Ciclo de Mejora en el Aula}

\section{Conexión con el proceso previo}

Este es el tercer curso que se implanta un Ciclo de mejora en la asignatura. El curso 2018-19 se implantó un Ciclo de mejora de 8 horas, que en general se desarrolló bien (Vargas, 2018). Los resultados de la evaluación mostraron que el ciclo funcionó y que los alumnos habían adquirido conocimiento sobre las cuestiones relevantes de la asignatura. El curso 2019-20, se amplió la extensión del mismo para que cubriese 19 horas. Un aspecto importante en este segundo ciclo era la importancia de usar preguntas diferentes a las de la preevaluación para evaluar el proceso de aprendizaje (Vargas, 2020).

El presente ciclo de mejora se diseñó para adaptarlo al primer bloque de la asignatura "Psicología de la Atención y de la Percepción" del Grado en Psicología de la Universidad de Sevilla y que cubra tanto las clases no presenciales como las presenciales. En concreto el ciclo abarcará todo el bloque de Psicología de la Percepción. Este ciclo de mejora comenzó el 6 de octubre y concluyó el 19 de noviembre con una extensión total de 21 horas distribuidas en grupo grande no presencial (14 horas) y grupo pequeño presencial (7 horas). El CIMA del curso anterior se centró en plantear problemas que guiaran la intervención, a la vez que servían para evaluar el conocimiento inicial del alumnado y poder adaptar el proceso enseñanza-aprendizaje al mismo. El nuevo CIMA para el Curso 2020-21 en la asignatura de Psicología de la atención y de la Percepción pretendía continuar en la misma línea incidiendo en el aprendizaje basado en problemas para fomentar el interés del alumnado. Aunque originalmente se planteó continuar Ciclos de Mejora en el Aula (2020). Experiencias de Innovación Docente de la US
Esta obra se distribuye con la licencia Creative Commons 
con el trabajo de estas cuestiones en diferentes grupos de tamaño creciente para potenciar la participación, así como el trabajo en grupo respetando las medidas de distanciamiento social actuales. De forma general, la principal característica del modelo metodológico que guía presente CIMA se basa en el concepto "Flipped classroom" o "Aula invertida" (Tucker, 2012).

\section{Mapa de contenidos}

El mapa de contenidos que vertebró el presente CIMA se desarrolla a continuación en la Figura 1.

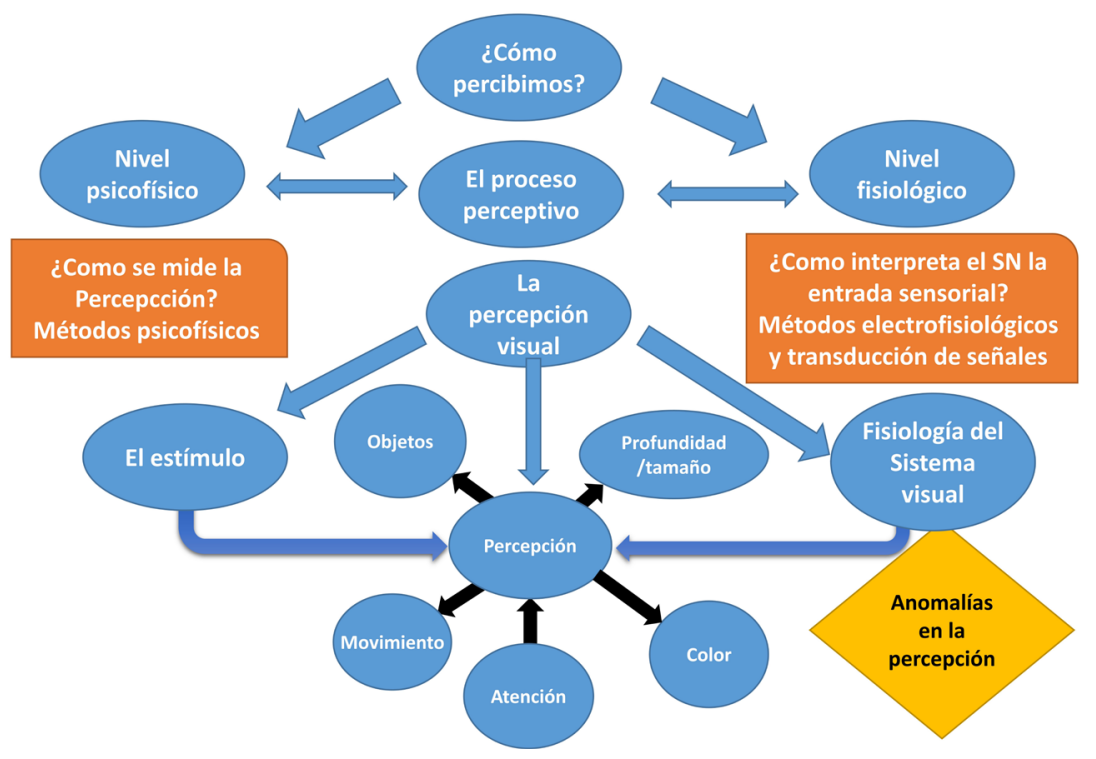

Figura 1. Mapa de contenidos de la asignatura. Los círculos azules corresponden a contenido conceptual, los cuadrados marrones indican contenido procedimental y los rombos naranjas indican contenido actitudinal o de valores.

Ciclos de Mejora en el Aula (2020). Experiencias de Innovación Docente de la US (i) Esta obra se distribuye con la licencia Creative Commons Reconocimiento-NoComercial-SinObraDerivada Internacional (CC BY-NC-ND 4.0.) 


\section{Actividades}

A continuación se exponen las diferentes actividades trabajadas en el CIMA:

Actividad 1. Sesión 1. Duración: 1 hora. Tipología: Presencial

Reflexión sobre los conceptos claves en psicología de la percepción.

Conceptos tratados:

1. ¿Qué es la Psicología?

2. ¿Qué es la percepción?

3. Lista otros procesos de los que se encargue la psicología

4. Realiza una clasificación de estos procesos de más importante a menos importante.

5. ¿Por qué crees que el estudio de la percepción puede ser importante para un psicólogo?

Actividad 2. Sesión 2. Duración: 2 horas. Tipología: telemática

Niveles en el estudio de la percepción

Conceptos tratados:

- Proceso perceptivo

6. Enumera los pasos que podemos encontrar en el proceso perceptivo

7. Define qué relaciones entre estos pasos se encarga de estudiar la psicofísica (nivel psicofísico).

8. Define qué relaciones entre estos pasos se encarga de estudiar la fisiología (Nivel fisiológico).

- Nivel de análisis psicofísico

Ciclos de Mejora en el Aula (2020). Experiencias de Innovación Docente de la US Esta obra se distribuye con la licencia Creative Commons 
9. ¿Cómo podemos cuantificar una respuesta perceptiva?

10. ¿Cuál es el resultado final de la percepción?

11. ¿Qué utilidad tiene el proceso perceptivo?

- Nivel de análisis fisiológico

12. Define las principales estructuras del ojo

13. Define los principales elementos de la retina

14. ¿Para qué sirve la fóvea?

15. ¿Por qué existe el punto ciego?

16. ¿Cuál es la ventaja de tener diferentes tipos de receptores en la retina?

17. ¿Por qué los piratas se suelen representar con un parche en un ojo?

18. ¿Qué efectos tiene el hecho de tener más de 120 millones de receptores en la retina, pero solo 1 millón de células ganglionares?

19. ¿Qué efectos explica la inhibición lateral?

Actividad 2. Sesión 3. Duración: 1 horas. Tipología: presencial

Puesta en común de los conceptos tratados en la sesión 2.

Actividad 3. Sesión 4. Duración: 2 horas. Tipología: telemática

Teorías para el estudio de la percepción

Conceptos tratados:

20. Diferencias entre estimulación distal y proximal

21. Correspondencia entre estimulación distal y proximal.

22. Diferencias teorías directas/cognitivas vs teorías indirectas/ecología perceptiva

Ciclos de Mejora en el Aula (2020). Experiencias de Innovación Docente de la US Esta obra se distribuye con la licencia Creative Commons 
23. Mecanismos compensatorios. ¿Qué son y cuál es su relación con las constancias perceptivas y con las ilusiones perceptivas?

24. ¿Cuál es el punto de partida de la percepción visual para las diferentes teorías?

Actividad 3. Sesión 5. Duración: 1 horas. Tipología: presencial

Puesta en común de los conceptos tratados en la sesión 4.

Actividad 4. Sesión 6. Duración: 2 horas. Tipología: telemática

Percepción de objetos

- Organización Perceptiva

25. Define los conceptos heurístico y algoritmo y pon un ejemplo de cada uno.

26. ¿Piensas que la percepción visual funciona de forma heurística o algorítmica? Fundamenta tu respuesta

27. Enumera los principales principios gestálticos de la percepción.

28. Pon un ejemplo donde más de uno de estos principios entren en conflicto.

- Segregación Perceptiva

29. ¿Qué son las figuras reversibles?

30. Enumera las diferencias entre figura-fondo

31. Enumera factores para la determinación de la figura en una escena visual

- Construcción de objetos

32. Principios de la teoría de integración de características

33. Diferencias entre procesamiento de información en serie-paralelo

34. ¿Qué es el procedimiento de búsqueda visual?

Ciclos de Mejora en el Aula (2020). Experiencias de Innovación Docente de la US Esta obra se distribuye con la licencia Creative Commons 
35. ¿Cómo se identifican las características básicas de los objetos según la TIC?

Actividad 4. Sesión 7. Duración: 1 horas. Tipología: presencial

Puesta en común de los conceptos tratados en la sesión 6.

Actividad 5. Sesión 8. Duración: 2 horas. Tipología: telemática

Percepción de tamaño y profundidad

36. ¿Cómo sabemos el tamaño que tiene un objeto?

37. ¿Cómo cambia nuestra percepción de un objeto en función de la distancia a la que se encuentra?

38. ¿Cuáles son los principales resultados del experimento de Holway y Boring (1941)?

39. ¿Cómo se forma una postimagen?

40. ¿Qué plantea la Ley de Emmert?

41. Razona por qué se produce una ilusión perceptiva cuando fallan los mecanismos compensatorios para la constancia del tamaño en una ilusión clásica

42. Enumera las claves de profundidad de origen fisiológico

43. Enumera las claves de profundidad de tipo pictórico

44. Enumera las claves de profundidad producidas por el movimiento

45. ¿Qué es la disparidad ocular?

46. ¿Qué es la estereopsia?

47. ¿Cuál es la relación entre disparidad ocular y horóptero?

Actividad 5. Sesión 9. Duración: 1 horas. Tipología: presencial

Ciclos de Mejora en el Aula (2020). Experiencias de Innovación Docente de la US Esta obra se distribuye con la licencia Creative Commons 

sión 6.

Puesta en común de los conceptos tratados en la se-

Actividad 6. Sesión 10. Duración: 2 horas. Tipología: telemática

Percepción del movimiento

48. Plantea como se percibe el movimiento desde la perspectiva indirecta de la percepción

49. Plantea como se percibe el movimiento desde la perspectiva directa de la percepción

50. En psicofísica, de qué factores depende la detección de movimiento.

51. ¿Qué es el movimiento estroboscópico?

52. Enumera ilusiones visuales de movimiento

53. ¿Qué elementos intervienen en la teoría de la descarga corolaria?

54. ¿Cómo explican el movimiento las teorías directas de la percepción?

Actividad 6. Sesión 11. Duración: 1 horas. Tipología: presencial

Puesta en común de los conceptos tratados en la sesión 10.

Actividad 7. Sesión 12. Duración: 2 horas. Tipología: telemática

Percepción del color

55. ¿Qué importancia/ventaja tiene la percepción de los colores?

56. ¿Cuál es la diferencia física entre dos colores?

Ciclos de Mejora en el Aula (2020). Experiencias de Innovación Docente de la US Esta obra se distribuye con la licencia Creative Commons 
57. ¿Cómo podemos describir un color? ¿Cuáles son las características del mismo?

58. ¿Cuál es el color resultante de mezclar dos colores? Pon ejemplos, ¿por qué surgen estos colores y no otros?

59. ¿Cuáles son las deficiencias de percepción del color? ¿Por qué ocurren? ¿Qué medidas se pueden tomar para minimizar su impacto en la vida cotidiana?

Actividad 7. Sesión 13. Duración: 1 horas. Tipología: presencial

Puesta en común de los conceptos tratados en la sesión 12.

Actividad 8. Sesión 13. Duración: 2 horas. Tipología: telemática

Puesta en común y evaluación de la experiencia.

\section{Modelo metodológico ideal y posible para el nuevo CIMA}

En el modelo metodológico se ha intentado plasmar las actividades planificadas para las 21 horas del ciclo de mejora adaptándolas a la organización de las clases. Para ello se ha dividido las tareas en función del grupo de trabajo. Para las sesiones de grupo grande (14 horas) que se realizan de forma telemática se han planificado 7 sesiones de trabajo sobre problemas de dos horas de duración. La dinámica de trabajo será parecida para cada bloque de problemas. En primer lugar, los alumnos trabajan los problemas de forma individual, posteriormente se comparten las respuestas para que los alumnos reflexionen sobre las distintas soluciones aportadas. A partir de aquí, tienen que elaborar de nuevo las soluciones, cambiando las que consideren. En la clase siguiente que fue de grupo pequeño y presencial, realizaremos una puesta en común y debate

Ciclos de Mejora en el Aula (2020). Experiencias de Innovación Docente de la US Esta obra se distribuye con la licencia Creative Commons 
sobre estos problemas. La última hora de grupo grande hicimos una puesta en común y evaluación de la experiencia. Los contenidos de tipo procedimental, actitudinal o de valores se trataron en todas las sesiones de forma transversal, aunque en las sesiones de grupo pequeño se trabajaron más específicamente este tipo de contenidos.

\section{Seguimiento de la evolución de los modelos mentales de los estudiantes}

Para el seguimiento de la evolución de los modelos mentales de los estudiantes se usó un sistema de evaluación de escalera de respuestas. Por una parte, al inicio de la actividad los alumnos rellenaron un cuestionario inicial sobre respuestas generales sobre psicología de la atención y de la percepción. Al final del ciclo, los alumnos respondieron a un cuestionario sobre los mismos conceptos que trabajaron en el primer cuestionario, pero con preguntas ligeramente diferentes. El cuestionario usado es el mismo que se usó en el curso anterior con la intención de poder comparar los resultados de un curso presencial con otro semipresencial.

\section{Cuestionario inicial}

1. ¿Cuál es la importancia del estudio de la percepción para un psicólogo?

2. ¿Cómo podemos cuantificar una respuesta perceptiva?

3. ¿Para qué sirven los diferentes receptores de la retina?

4. ¿Cómo conseguimos crear una representación interna de lo que percibimos externamente y qué características tiene?

5. ¿Cómo sabemos de qué tamaño es un objeto? ¿Qué ocurre si cometemos un error de cálculo de tamaño?

Ciclos de Mejora en el Aula (2020). Experiencias de Innovación Docente de la US Esta obra se distribuye con la licencia Creative Commons 
6. En una escena visual, podemos diferenciar figura y fondo. Define los conceptos y resalta las diferencias.

7. ¿Cómo sabemos el tamaño que tiene un objeto?

8. ¿Para qué sirve y cómo funciona la disparidad ocular? Es decir, la diferencia entre lo que vemos por un ojo y por el otro.

9. ¿De qué factores depende que detectemos que un objeto está en movimiento?

10. ¿Qué importancia/ventaja tiene la percepción de los colores?

11. ¿Cuál es la diferencia física entre dos colores?

12. ¿Cuáles son las deficiencias de percepción del color? ¿Por qué ocurren? ¿Qué medidas se pueden tomar para minimizar su impacto en la vida cotidiana?

El cuestionario final era una versión ligeramente diferente, en el que las preguntas estaban reformuladas y cambiadas de orden, pero se mantenían los mismos conceptos.

\section{Valoración de la docencia}

Para la evaluación de la experiencia se programó la Actividad 8 que tuvo lugar durante la Sesión 13 con una duración de 2 hora. En esta sesión se puso en común la valoración de los elementos docentes que han guiado esta experiencia. Desde la estructura de trabajo en las sesiones hasta la pertinencia de contenidos o el papel del docente. Para el presente trabajo se han evaluado los cuestionarios del grupo pequeño más numeroso que contaba con 16 alumnos de los cuales 14 cumplimentaron el cuestionario inicial y el final.

Ciclos de Mejora en el Aula (2020). Experiencias de Innovación Docente de la US Esta obra se distribuye con la licencia Creative Commons 


\section{Aplicación del CIMA}

Este curso ha tenido la particularidad de que la mayor parte de las clases se han impartido de forma no presencial debido a la situación creada por la pandemia de COVID-19. Las clases no presenciales estaban dirigidas a todo el grupo que eran 52 alumnos y en las presenciales el grupo se dividía en 4 con lo que estos grupos presenciales contaban con una media de 13 alumnos. Una diferencia importante con años anteriores es que la asistencia a cada una de las sesiones era prácticamente completa. El hecho de tener menos horas de docencia que otros años probablemente ha propiciado este aumento significativo de la asistencia. Por otra parte, las medidas de distanciamiento impuestas por la situación de pandemia han dificultado el trabajo en grupo.

En la siguiente tabla se recoge el nivel de elaboración de las respuestas al cuestionario inicial y final. Se han establecido cuatro niveles de elaboración de respuesta en cada pregunta. En la tabla se recoge el porcentaje de respuestas de cada nivel en el cuestionario previo y en el final, así como la variación porcentual en cada uno. Como puede observarse, en todas las preguntas ha habido un avance significativo en la prueba final.

Tabla 1. Resultados de los cuestionarios previo y final y porcentaje de variación en cada nivel de respuesta

\begin{tabular}{|l|l|l|l|l|}
\hline $\begin{array}{l}\text { Respuesta } \\
\text { Preguntal }\end{array}$ & $\begin{array}{l}\text { Nivel de } \\
\text { respuesta }\end{array}$ & \% Pretest & \% PostTest & \% Variación \\
\hline $\mathbf{1}$ & 0 & 50 & 0 & -50 \\
& 1 & 50 & 21,4 & $-28,6$ \\
& 2 & 0 & 57,2 & $+57,2$ \\
& 3 & 0 & 21,4 & $+21,4$ \\
\hline $\mathbf{2}$ & 0 & 42,9 & 0 & $-42,9$ \\
& 1 & 57,1 & 28,6 & $-28,5$ \\
& 2 & 0 & 50 & +50 \\
& 3 & 0 & 21,4 & $+21,4$ \\
\hline
\end{tabular}

Ciclos de Mejora en el Aula (2020). Experiencias de Innovación Docente de la US Esta obra se distribuye con la licencia Creative Commons 


\begin{tabular}{|c|c|c|c|c|}
\hline 3 & $\begin{array}{l}0 \\
1 \\
2 \\
3\end{array}$ & $\begin{array}{l}28,6 \\
42,8 \\
28,6 \\
0\end{array}$ & $\begin{array}{l}14,3 \\
7,1 \\
57,1 \\
21,5\end{array}$ & $\begin{array}{l}-14,3 \\
-35,7 \\
+28,6 \\
+21,5\end{array}$ \\
\hline 4 & $\begin{array}{l}0 \\
1 \\
2 \\
3\end{array}$ & $\begin{array}{l}7,1 \\
64,4 \\
21,4 \\
7,1\end{array}$ & $\begin{array}{l}0 \\
14,3 \\
21,4 \\
64,4\end{array}$ & $\begin{array}{l}-7,1 \\
-50,1 \\
0 \\
+57,3\end{array}$ \\
\hline 5 & $\begin{array}{l}0 \\
1 \\
2 \\
3\end{array}$ & $\begin{array}{l}14,3 \\
35,7 \\
50 \\
0\end{array}$ & $\begin{array}{l}0 \\
14,3 \\
42,9 \\
42,9\end{array}$ & $\begin{array}{l}-14,3 \\
-21,4 \\
-7,1 \\
42,8\end{array}$ \\
\hline 6 & $\begin{array}{l}0 \\
1 \\
2 \\
3\end{array}$ & $\begin{array}{l}0 \\
78,6 \\
21,4 \\
0\end{array}$ & $\begin{array}{l}0 \\
50 \\
28,6 \\
21,4\end{array}$ & $\begin{array}{l}0 \\
-28,6 \\
+7,2 \\
+21,4\end{array}$ \\
\hline 7 & $\begin{array}{l}0 \\
1 \\
2 \\
3\end{array}$ & $\begin{array}{l}0 \\
42,9 \\
57,1 \\
0\end{array}$ & $\begin{array}{l}0 \\
7,1 \\
21,4 \\
71,5\end{array}$ & $\begin{array}{l}0 \\
-35,8 \\
-35,7 \\
+71,5\end{array}$ \\
\hline 8 & $\begin{array}{l}0 \\
1 \\
2 \\
3\end{array}$ & $\begin{array}{l}14,3 \\
64,3 \\
21,4 \\
0\end{array}$ & $\begin{array}{l}0 \\
21,4 \\
64,3 \\
14,3\end{array}$ & $\begin{array}{l}-14,3 \\
-42,9 \\
+42,9 \\
+14,3\end{array}$ \\
\hline 9 & $\begin{array}{l}0 \\
1 \\
2 \\
3\end{array}$ & $\begin{array}{l}7,1 \\
35,7 \\
57,2 \\
0\end{array}$ & $\begin{array}{l}0 \\
21,4 \\
42,9 \\
35,7\end{array}$ & $\begin{array}{l}-7,1 \\
-14,3 \\
-14,3 \\
+35,7\end{array}$ \\
\hline 10 & $\begin{array}{l}0 \\
1 \\
2 \\
3\end{array}$ & $\begin{array}{l}0 \\
50 \\
42,9 \\
7,1\end{array}$ & $\begin{array}{l}0 \\
0 \\
64,3 \\
35,7\end{array}$ & $\begin{array}{l}0 \\
-50 \\
+21,4 \\
+28,6\end{array}$ \\
\hline 11 & $\begin{array}{l}0 \\
1 \\
2 \\
3\end{array}$ & $\begin{array}{l}50 \\
28,6 \\
14,4 \\
7,1\end{array}$ & $\begin{array}{l}14,3 \\
7,1 \\
42,9 \\
35,7\end{array}$ & $\begin{array}{l}-35,7 \\
-21,5 \\
+28,6 \\
+28,6\end{array}$ \\
\hline
\end{tabular}

Para simplificar estos datos y poder compararlos con los datos del curso previo en el que la docencia era exclusivamente presencia hemos calculado un parámetro por cada pregunta en el que se suman las respuestas de nivel 1 con el doble de las respuestas de nivel 2 y el triple de las

Ciclos de Mejora en el Aula (2020). Experiencias de Innovación Docente de la US Esta obra se distribuye con la licencia Creative Commons 
de nivel 3. El cálculo de este índice arroja un valor de 113 para el cuestionario previo y un valor de 212,4 para el cuestionario final. Analizando los datos del curso previo, los valores para el cuestionario previo y final son 122,4 y 217 , respectivamente. Estos datos indican que en ambos cursos hubo una mejora significativa en los resultados de cuestionario final respecto al cuestionario inicial. Se observa un cierto paralelismo en los resultados de ambos cursos con una ligera mejora en el cuestionario previo del curso pasado que se diluye con el proceso de aprendizaje. Este resultado estaría indicando que no ha habido un efecto negativo debido al menor número de clases y a la situación de semipresencialidad.

\section{Evaluación del CIMA}

La aplicación de este ciclo de mejora ha estado condicionada por la excepcionalidad que supone impartir docencia en tiempos de pandemia en una Universidad presencial. Esta particularidad ha provocado que se tengan que hacer numerosos ajustes. Uno de ellos ha sido mantener un sistema de evaluación similar al usado en el curso anterior en una situación de docencia presencial. Como aspectos positivos del presente ciclo se pueden destacar los siguientes:

- El desarrollo de un sistema de trabajo continuo que permite tener una relación más fluida con el alumnado a pesar de las pocas clases presenciales.

- Como factores potencialmente facilitadores de este proceso podríamos señalar que los grupos presenciales estaban compuestos por unos 12-16 alumnos con lo que se favorece el trabajo directo con ellos.

- Se reafirma la idea propuesta en el CIMA del curso previo de que el hecho de que los estudiantes trabajen los conceptos de la asignatura de forma autónoma y, posteriormente en pequeños grupos, facilita que adquieran los conocimientos generales de la asignatura.

Ciclos de Mejora en el Aula (2020). Experiencias de Innovación Docente de la US Esta obra se distribuye con la licencia Creative Commons 
Por otra parte, hay una serie de aspectos que podrían mejorarse, eliminarse 0 , al menos, evaluarlos para determinar su pertinencia en el ciclo.

- El hecho de que el grupo tuviese tan poca relación personal ha impactado en la dinámica de debates. Si esta situación se prolongase en el tiempo, es posible que todos nos vayamos adaptando a la interacción en situaciones de no presencialidad.

- Al iniciar este ciclo se tuvieron que tomar decisiones para adaptarlo a la nueva situación. Una de ellas fue usar los instrumentos del curso pasado para poder hacer una comparativa. Sin embargo, en el CIMA del curso pasado se detectaron una serie de problemas en relación con preguntas de trabajo redundantes y algunos aspectos clave de la asignatura que no se tocaban suficientemente.

A modo de conclusión, el principal elemento positivo de este ciclo, es el hecho de que podemos tomar conciencia sobre los procesos de enseñanza y los contenidos a impartir teniendo en cuenta el punto de partida de los estudiantes (Finkel, 2008) y adaptarlos a un escenario de semipresencialidad, como es el caso, o de no presencialidad si se diese esta circunstancia. Respecto al ciclo de mejora previo, en este hemos tenido que hacer un esfuerzo por trasladar una metodología de trabajo con mucho dinamismo físico a un escenario de distanciamiento físico. A nivel personal, el hecho de tener que adaptarme a estos cambios, ha hecho que afronte de manera más activa la adaptación de mi docencia a un escenario de no presencialidad.

Ciclos de Mejora en el Aula (2020). Experiencias de Innovación Docente de la US Esta obra se distribuye con la licencia Creative Commons 
Palabras clave: Psicología de la Percepción, Psicología de la Atención, Psicología, Docencia universitaria y Experimentación docente universitaria.

Keywords: Perception Psychology, Attention Psychology, Psychology, University Teaching and University Teaching Experimentation.

\section{Bibliografía}

Bain, K. (2007). Lo que hacen los mejores profesores universitarios, Valencia: Publicaciones Universidad de Valencia.

Finkel, D. (2008). Dar clase con la boca cerrada. Valencia: Publicaciones Universidad de Valencia.

Harland, T. (2017). Enseñanza universitaria. Una guía introductoria. Madrid: Morata.

Porlán, R. (2017). Enseñanza Universitaria. Cómo mejorarla. Madrid: Editorial Morata.

Tucker, B. (2012). The flipped classroom. Education Next, 12, 82-83

Vargas, J.P. (2018). Ciclo de Mejora Docente en la asignatura de Psicología de la Atención de la Percepción del Grado en Psicología. En R. Porlán y E. Navarro-Medina (Coords.), V Jornadas de Docencia Universitaria (págs. 1773-1790). Sevilla: Instituto de Ciencias de la Educación de la Universidad de Sevilla. D.O.I.: http://dx.doi. org/10.12795/JDU.2018.i01.100

Vargas, J.P. (2020). Aplicación de un Ciclo de Mejora en el aula (CIMA) en la asignatura de Psicología de la Percepción. En E. Navarro Medina. y R. Porlán Ariza (Eds). Ciclos de mejora en el aula. Año 2019. Experiencias de innovación docente de la Universidad de Sevilla. Sevilla: Editorial Universidad de Sevilla.

Ciclos de Mejora en el Aula (2020). Experiencias de Innovación Docente de la US Esta obra se distribuye con la licencia Creative Commons 